\title{
THE NUMBER OF CONTINUA
}

\author{
F. W. LOZIER AND R. H. MARTY
}

\begin{abstract}
It is shown there are precisely $2^{n}$ topologically distinct continua of weight $n$ and power $m$ where $p \leqq n \leqq m$ and $p$ is the smallest cardinal for which there is a continuum of power $m$ and weight $p$. In particular, there are precisely $2^{m}$ topologically distinct continua of power $m$.
\end{abstract}

All spaces considered are assumed to be Hausdorff and all cardinals infinite. A continuum is a compact connected space. It follows from a classical result of $\mathrm{P}$. Alexandroff and P. Urysohn $([1, \mathrm{p} .105]$ : The weight of a compact space never exceeds its power) that there are at most $2^{m}$ topologically distinct compact spaces of power $m$. (There are just $2^{m}$ collections, of cardinality at most $m$, of subsets of a set of cardinality $m$.) The following sharper result will be useful.

Proposition 1. There are at most $2^{n}$ topologically distinct compact spaces of weight $n$.

Proof. Suppose $\left\{X_{\xi}: \xi \in \Xi\right\}$ is a collection of topologically distinct compact spaces of weight $n$. For each $\xi \in \Xi$, let $\mathscr{B}_{\xi}$ be a base of cardinality $n$ for $X_{\xi}$. For each pair $U, V \in \mathscr{B}_{\xi}$ with $\bar{U} \cap \bar{V}=\varnothing$, let $f_{U, V}$ be an element of $C\left(X_{\xi}\right)$ such that $f_{U, V} \mid U=1$ and $f_{U, V} \mid V=0$. Let $\mathscr{F}_{\xi}$ denote the subset of $C\left(X_{\xi}\right)$ consisting of all these $f_{U, V}$ 's together with all constant functions $f(x)=r$ with $r$ rational. Then card $\mathscr{F}_{\xi} \leqq n$ and, by the Stone-Weierstrass Theorem, the smallest closed subring of $C\left(X_{\xi}\right)$ containing $\mathscr{F}_{\xi}$ is $C\left(X_{\xi}\right)$, where $C\left(X_{\xi}\right)$ is given the usual metric.

Now let $Z$ be a fixed discrete space of power $n$ and let $C^{*}(Z)$ have the usual metric. For each $\xi \in \Xi$, choose $f_{\xi}: Z \rightarrow X_{\xi}$ such that $f_{\xi}[Z]$ is dense in $X_{\xi}$, and let $F_{\xi}: C\left(X_{\xi}\right) \rightarrow C^{*}(Z)$ be the induced map of $f_{\xi}$; i.e., such that $F_{\xi}(f)=f \circ f_{\xi}$ for every $f \in C\left(X_{\xi}\right)$. Then each $F_{\xi}$ is a ring isomorphism so that, for $\xi \neq \xi^{\prime}, F_{\xi}\left[C\left(X_{\xi}\right)\right]$ and $F_{\xi^{\prime}}\left[C\left(X_{\xi^{\prime}}\right)\right]$ are nonisomorphic, and hence distinct, subrings of $C^{*}(Z)$. Furthermore, each $F_{\xi}$ is an isometry; in particular, since $C\left(X_{\xi}\right)$ is complete, $F_{\xi}\left[C\left(X_{\xi}\right)\right]$ is a closed subspace of

Received by the editors October 4, 1972.

AMS (MOS) subject classifications (1970). Primary 54A25, 54F15; Secondary 54A10, 54D05.

Key words and phrases. Continuum, weight, power, long line.

(c) American Mathematical Society 1973 
$C^{*}(Z)$. Thus the smallest closed subring of $C^{*}(Z)$ containing $F_{\xi}\left[\mathscr{F}_{\xi}\right]$ is $F_{\xi}\left[C\left(X_{\xi}\right)\right]$ for every $\xi \in \Xi$. Thus for $\xi \neq \xi^{\prime}, F_{\xi}\left[\mathscr{F}_{\xi}\right] \neq F_{\xi^{\prime}}\left[\mathscr{F}_{\xi^{\prime}}\right]$. Consequently, $\left\{F_{\xi}\left[\mathscr{F}_{\xi}\right]: \xi \in \Xi\right\}$ is a collection of distinct subsets of $C^{*}(Z)$ of cardinality at most $n$. But $C^{*}(Z)$ has cardinality $2^{n}$ and hence at most $2^{n}$ subsets of cardinality at most $n$. Thus card $\Xi \leqq 2^{n}$.

Proposition 2. For every cardinal $m \geqq 2^{\mathrm{N}_{0}}$, there are $2^{m}$ topologically distinct continua of power $m$ and weight $m$.

Proof. Let $L$ denote the long line constructed on the set $S$ of all ordinals $\beta \leqq \omega(m)$, the initial ordinal of cardinality $m$. We regard $S$ as a subset of $L$. For each $\beta \in S$, let $\left\langle X_{\beta}, x_{\beta}\right\rangle$ be either $\left\langle I^{2},(0,0)\right\rangle$ or $\left\langle I^{3},(0,0,0)\right\rangle$, where $I=[0,1] \subset R$. Let $X$ be the space obtained by attaching each $X_{\beta}$ to $L$ by identifying $x_{\beta} \in X_{\beta}$ with $\beta \in S$, and weakening the usual quotient topology by requiring that any neighborhood of a limit ordinal $\gamma \in S$ contains $\bigcup\left\{X_{\beta}: \alpha<\beta<\gamma\right\}$ for some $\alpha<\gamma$. Then $X$ is a continuum of power $m$ and weight $m$.

Now suppose $X^{\prime}$ were another such space constructed in the same way but with a conceivably different choice of the $\left\langle X_{\beta}^{\prime}, x_{\beta}^{\prime}\right\rangle$ 's, and suppose $f: X \rightarrow X^{\prime}$ were an onto homeomorphism. Let $Y$ denote the set of all points of $X$ at which the dimension is 1 ; then $L=\bar{Y}$ and $S=\bar{Y} \cap(X-Y)$. Similarly, $L^{\prime}=\bar{Y}^{\prime}$ and $S^{\prime}=\bar{Y}^{\prime} \cap\left(X^{\prime}-Y^{\prime}\right)$ in $X^{\prime}$. It is immediate that $f[Y]=Y^{\prime}$, and hence $f[L]=L^{\prime}$ and $f[S]=S^{\prime}$. But then $f \mid L$ is monotone, so that $f \mid S$ is order-preserving and hence the identity. Now consider the subspace $Z=\bigcup\left\{X_{\beta}: \beta \in S\right\}$ of $X$, which is the disjoint union of the connected subspaces $X_{\beta}$, and the analogous subspace $Z^{\prime}$ of $X^{\prime}$. Because $Z=(X-L) \cup S$ and $Z^{\prime}=\left(X^{\prime}-L^{\prime}\right) \cup S^{\prime}$, it follows that $f[Z]=Z^{\prime}$; in particular, $f$ maps each $X_{\beta}$ onto some $X_{\beta}^{\prime}$. Because $f \mid S$ is the identity, it follows that $f\left[X_{\beta}\right]=X_{\beta}^{\prime}$ for every $\beta \in S$. Therefore, since $I^{2}$ and $I^{3}$ are not homeomorphic, it follows that $X_{\beta}^{\prime}=X_{\beta}$ for every $\beta \in S$.

Finally, since there are $2^{m}$ different ways to choose the $X_{\beta}$ 's there are $2^{m}$ topologically distinct continua of power $m$ and weight $m$.

PRoposition 3. For every cardinal $m \geqq 2^{N_{0}}$, let $p$ be the smallest cardinal for which there is a continuum of power $m$ and weight $p$. Then for every cardinal $n$ with $p \leqq n \leqq m$, there are $2^{n}$ topologically distinct continua of power $m$ and weight $n$.

Proof. Let $K$ be a continuum of power $m$ and weight $p$. For any cardinal $n$ with $p \leqq n \leqq m$, let $L$ be the long line constructed on the set of all ordinals $\beta \leqq \omega(n)$. Construct $X$ as in Proposition 2; but take $X_{0}$ to be $K \times I^{2}$ and take $X_{\beta}$, for $\beta>0$, to be either $I^{2}$ or $I^{3}$. The argument is then similar to Proposition 2. 
RemarKs. For the case $m=2^{\aleph_{0}}$, the requirement that $X_{0}=K \times I^{2}$ can be dispensed with. Furthermore, for the case $m=2^{\aleph_{0}}, n=\aleph_{0}$, all of the constructed continua can be embedded in the plane by choosing $X_{\beta}$ to be either $I^{2}$ or an annulus. If $m=2^{q}$ for some $q$, then $p$ is simply the smallest such $q$; for then $I^{p}$ is a continuum of power $m$ and weight $p$. In particular, if we assume the Generalized Continuum Hypothesis, then the only continua of power $m$ other than those of weight $m$ are (for nonlimit cardinals $m$ ) the $2^{n}$ topologically distinct continua of weight $n$ where $2^{n}=m$.

The authors wish to express their gratitude to S. Mrowka and the referee for their helpful suggestions.

\section{REFERENCES}

1. R. Engelking, Outline of general topology, North-Holland, Amsterdam; Interscience, New York, 1968. MR 37 \#5836.

2. L. Gillman and M. Jerison, Rings of continuous functions, University Series in Higher Math., Van Nostrand, Princeton, N.J., 1960. MR 22 \#6994.

Department of Mathematics, Cleveland State University, Cleveland, Ohio 44115 